Pramāṇa - J. Phys., Vol.30, No. 1, January 1988, pp. 29-33. (C) Printed in India.

\title{
A simple apparatus for the measurement of thermoelectric power in the temperature range $4 \cdot 2-300 \mathrm{~K}$
}

\author{
K P RAJEEV and N Y VASANTHACHARYA \\ Department of Physics, Indian Institute of Science, Bangalore 560012 \\ MS received 24 July 1987
}

\begin{abstract}
A simple apparatus to measure the absolute thermoelectric power of solids in the temperature range $4 \cdot 2-300 \mathrm{~K}$ is described. The cryostat and the associated instrumentation is simple to operate. Representative data of measurements on metallic wire and pressed pellets are given. An accuracy of better than $10 \%$ in absolute thermopower can be obtained in this apparatus.
\end{abstract}

Keywords. Thermoelectric power; low-temperature.

PACS Nos $06 \cdot 60 ; 72 \cdot 15 ; 72 \cdot 20$

\section{Introduction}

The Seebeck coefficient is an extremely sensitive property whose study as a function of temperature would give valuable information on the electronic structure of the system under study. The Seebeck coefficient is defined in a simple way as follows

$$
S=\lim _{\Delta T \rightarrow 0} \Delta E / \Delta T
$$

where $E$ is the differential voltage developed across the sample for a temperature difference $T$.

Thermoelectric power (TEP) can be measured using the differential technique wherein we create a temperature gradient across the sample and measure the voltage developed between the hot and cold ends of the thermocouple formed with a reference material. With the cold end of the sample connected to the positive of the measuring instrument the sign of the voltage developed gives the sign of the thermopower difference between the sample and the reference material. We describe in this paper a simple apparatus developed in our laboratory to measure thermoelectric power in the temperature range $4 \cdot 2-300 \mathrm{~K}$. There are many descriptions of TEP measurement in the literature (Eckland and Mabatah 1977; Wieder 1979). The technique that we have developed uses very simple instrumentation and it is easier operationally than many of the reported techniques. The purpose of the present paper is to provide necessary experimental details which are generally absent in other papers. It has been developed mainly for polycrystalline samples whose thermal conductance is not too high. A slight modification will also allow it to be used with other type of samples like a metallic strip or a metallic wire. We give below a description of the cryostat and the method of measurement. Some representative data taken using the apparatus are also shown. 


\section{Cryostat}

The schematic diagram of the cryostat is shown in figure 1. It essentially contains a high conductivity copper plate whose temperature can be controlled by an electronic controller. The base plate is attached to the copper shield using a thin stainless steel rod which is in turn brazed to the top flange of the cryostat. A $50 \mathrm{ohm}$ manganin heater (total length $2.5 \mathrm{~m}$ ) wound around the base plate and attached by low temperature varnish is used for temperature variation. All the electrical leads are thermally anchored to the shield. This whole set-up is housed in a cryostat which is immersed in the cryogenic liquid. The sample (typical diameter $13 \mathrm{~mm}$, thickness $3-5 \mathrm{~mm}$ ) whose thermopower is to be measured is held tightly between two polished copper discs on the base plate using a glass epoxy plate with brass screws as shown in the inset of figure 1 . The bottom copper disc is electrically insulated using a very thin cigarette paper attached with low temperature varnish. A small carbon resistor is used as heater on the top copper disc to create a temperature gradient. A differential gold-chromel thermocouple attached to the copper discs is used to measure the temperature difference between the hot and cold ends. The two chromel leads of the thermocouple terminate on the base plate. From there a pair of copper leads are taken to the voltmeter. Care is taken to ensure that the sample voltage leads and the thermocouple voltage leads are soldered using only low-thermal solder to minimize spurious voltages. Only shielded cables are used outside the cryostat. Calibrated platinum and germanium thermometers attached to the base plate are used for measuring the sample temperature. The temperature difference and the thermoelectric voltage developed across the sample are measured using a Keithley microvoltmeter (model 155) and a nanovolt meter (model 181) respectively. Both these instruments give analog outputs. The analog outputs (which are in the volt range) are plotted using

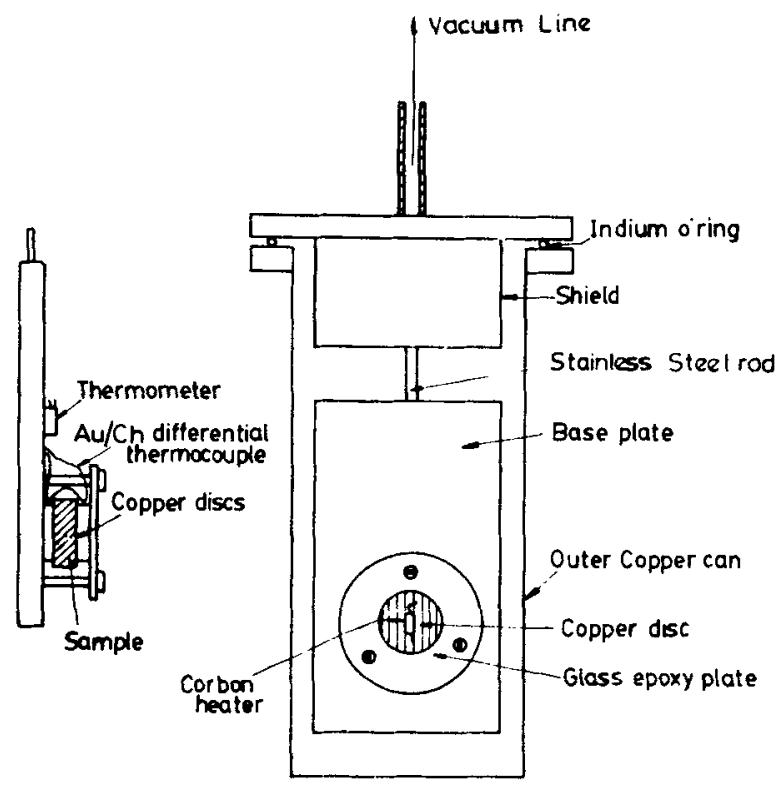

Figure 1. Schematic diagram of the thermopower cell and the outer can. 


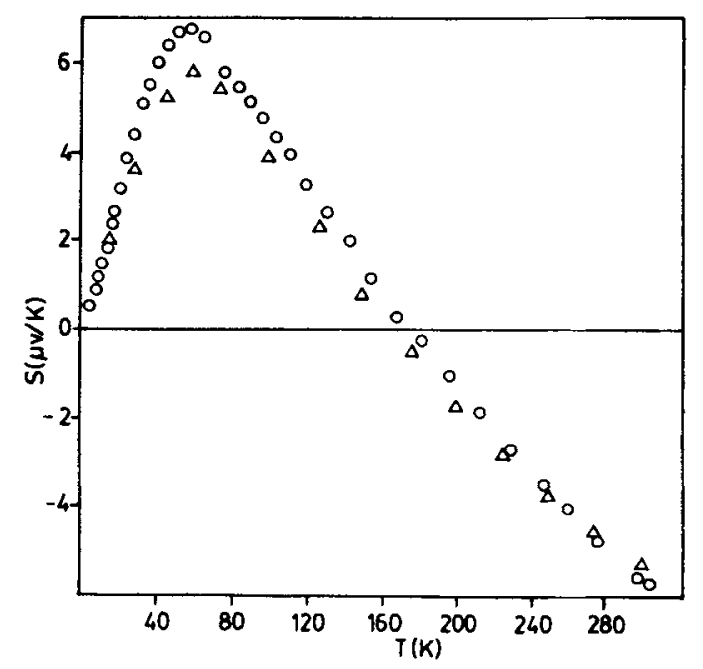

Figure 2. Absolute thermopower of platinum; $\bigcirc$-present data, $\triangle$-Huebner 1965.

an $\mathrm{X}-\mathrm{Y}$ recorder or recorded using a microprocessor. For temperature measurement and control a commercial 4-terminal bridge was used with a home-made PID controller (Mukhopadhyay and Raychaudhuri 1986). Generally the temperature control is within $0.01 \mathrm{~K}$ for temperatures less than $30 \mathrm{~K}$ and within $0.05 \mathrm{~K}$ for temperatures less than $100 \mathrm{~K}$ and within $0 \cdot 2 \mathrm{~K}$ up to $300 \mathrm{~K}$.

\section{Method of data acquisition}

To begin with the base temperature is stabilized at the temperature of interest and a small amount of power is applied to the top copper disc using the heater attached to it (typically $20 \mathrm{~mW}$ to $200 \mathrm{~mW}$ depending on the temperature and the thermal conductance of the sample). The temperature of the top copper disc increases steadily and consequently a small voltage will be developed across the hot and cold ends. The temperature difference was typically $0.5 \mathrm{~K}$ to $5 \mathrm{~K}$ depending on the temperature. A plot of these two voltages generally gives a straight line. If the heater power is sufficiently small so that the base temperature does not drift or $\Delta T / T$ is small the curve traces back in the reverse direction if the system is allowed to relax by switching off the heater power. The slope of the straight line thus traced is used to find out the Seebeck coefficient. In many situations we find that an acceptable straight line is not obtained or the heating or cocling curves show hysteresis. We found that such situations can come from the following reasons:

(1) base temperature is not properly controlled; (2) $\Delta T$ is too small (typically $\Delta T / T<1 \%$ gives problem); (3) bad thermal contact between sample and copper discs and also between thermocouple junctions and copper discs; (4) widely different equilibriation times for the sample and the thermocouple gives rise to hysteresis behaviour if the heating rate is too fast compared to the larger of the time constants (the heating rates used by us are as follows at $5 \mathrm{~K} 1 \mathrm{~K} / \mathrm{s}$, at $40 \mathrm{~K} 0 \cdot 1 \mathrm{~K} / \mathrm{s}$ and at $300 \mathrm{~K}$ $0.01 \mathrm{~K} / \mathrm{s}$ ). 
The slope of the heating (or cooling) curve gives the quantity $V_{\mathrm{S}-\mathrm{Cu}} / V_{\mathrm{Au}-\mathrm{CH}}\left(=S^{\prime}\right)$ where $V_{\mathrm{S}-\mathrm{Cu}}$ is the voltage developed across the $\mathrm{Cu}$-Sample-Cu thermocouple for a corresponding voltage $V_{\mathrm{Au}-\mathrm{Ch}}$ developed across the chromel-Au(Fe)-chromel couple. To obtain $S_{\mathrm{S}-\mathrm{Cu}}$ we convert $V_{\mathrm{Au}-\mathrm{Ch}}$ to a corresponding $\Delta T$ at the measuring temperature $T+\Delta T / 2$ where $T$ is the base temperature. This is done by using $S_{\mathrm{Au}-\mathrm{Ch}}$ from tabulated data (White 1979) and simple quadratic interpolation. To $S_{\mathrm{S}-\mathrm{Cu}}$, the absolute $S_{\mathrm{Cu}}$ is added to get $S_{\mathrm{S}}$, the absolute thermopower of the sample. $S_{\mathrm{Cu}}$ has been obtained from reported data (Dugdale 1977).

\section{Results}

Performance of the apparatus was tested by measuring TEP of platinum in the range $5-300 \mathrm{~K}$. For this purpose a length of platinum wire (length $4 \mathrm{~cm}$, dia $0.3 \mathrm{~mm}$ ) was wound across a fused quartz disc (dia $12 \mathrm{~mm}$, thickness $4 \mathrm{~mm}$ ) and it was placed between the copper discs. Figure 2 shows the absolute TEP of platinum measured by us and the literature value (Huebner 1965). We are routinely using this apparatus to measure TEP of perovskites showing metal-insulator transition. Recently the same apparatus has been used to measure first-ever reported thermopower of high $T_{c}$ oxides (Raychaudhuri et al 1987). The data are shown in the figure 3. From the series of measurements we can say that the absolute value of data are correct to $0.5 \mu \mathrm{V} / \mathrm{K}$. The reproducibility is also of the same order. Surface contamination sometimes create problems for nonmetallic samples. To check the effect of surface contamination in pressed pellets, we have taken data after scratching the surfaces of sample and copper discs a few times with emery paper. In figure 4 we show the results of such an experiment. It is found that the reproducibility is within $10 \%$.

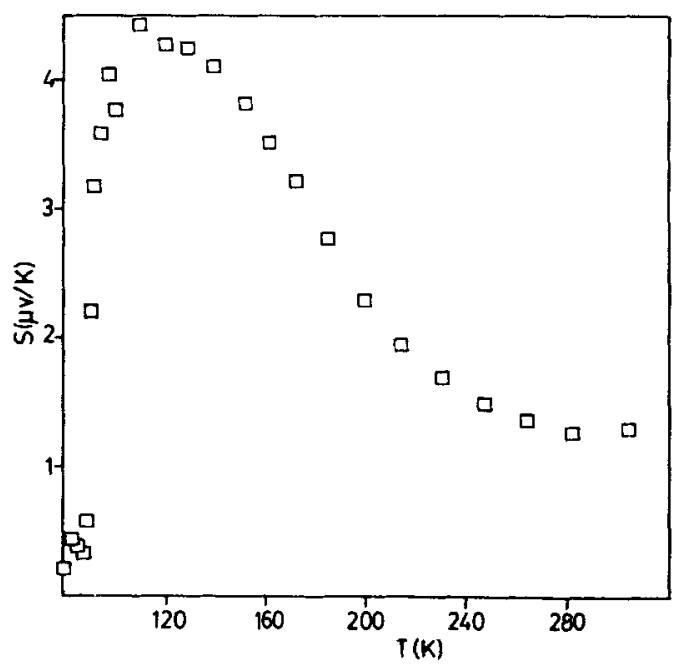

Figure 3. Absolute value of thermopower of recently discovered high $T_{\mathrm{c}}$ superconductor $\mathrm{YBa}_{2} \mathrm{Cu}_{3} \mathrm{O}_{7-\delta}$. 


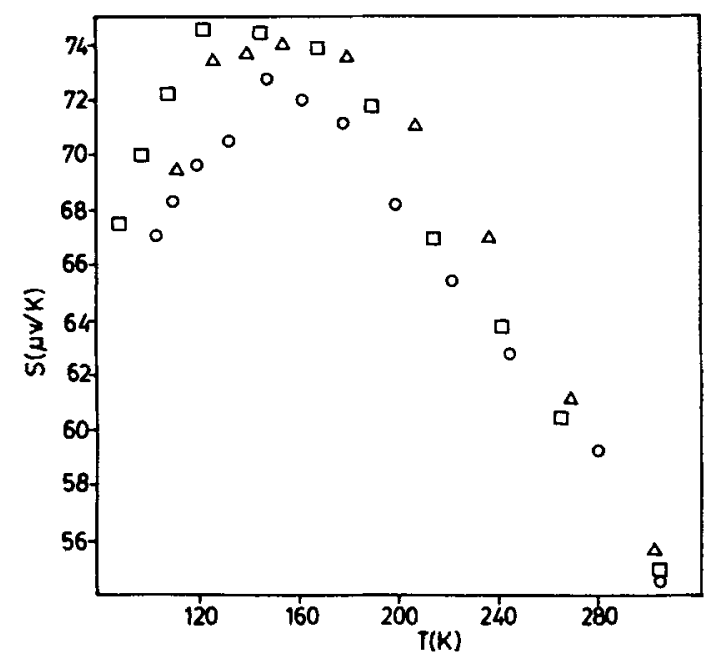

Figure 4. Thermopower of $\mathrm{LaNi}_{0 \cdot 25} \mathrm{Co}_{0.75} \mathrm{O}_{3}$ perovskite oxide; $\triangle$-dirty sample and sample holder, $\square$-clean sample and dirty sample holder, $\bigcirc$-clean sample and sample holder.

\section{Conclusion}

We have described here a simple apparatus of moderate to good accuracy for measuring TEP in the range $4 \cdot 2-300 \mathrm{~K}$. The apparatus is simple to use and easily accessible to microprocessor data acquisition. The necessary details have been given here and for further details the authors can be contacted.

\section{Acknowledgements}

The authors are extremely grateful to Dr A K Raychaudhuri for his help, encouragement and fruitful discussions. They would also like to thank the Department of Science and Technology for the financial support.

\section{References}

Dugdale J S 1977 Electrical properties of metals and alloys (London: Edward Arnold)

Eckland P C and Mabatah A K 1977 Ret. Sci. Instrum. 48775

Hucbner R P 1965 Phys. Rer. 140 A 1834

Mukhopadhyay P K and Raychaudhuri A K 1986 J. Phys. E19 792

Raychaudhuri A K, Sreedhar K, Rajeev K P, Mohan Ram R A, Ganguly P and Rao C N R 1987 Philos. Mag. Lett. (in print)

Wieder H H 1979 Laboratory notes on electrical and galvanomagnetic measurements (Amsterdarn: Elsevier) White G K (1979) Experimental techniques in low-temperature physics (Oxford: Clarendon) 